co-factor in methionine synthatase and methylmalonyl-CoA-mutase, a deficiency leading to the accumulation of homocysteine and methylmalonate in the plasma.

\title{
CSF NEOPTERIN LEVELS IN FEBRILE CONVULSIONS
}

Neopterin, a marker for activation of the cellular immune system, and interferon-gamma were measured in the cerebrospinal fluid of 40 infants and young children (ages 0.75 to 4.6 years) admitted with fever and/or convulsions to Nippon Medical School 2nd Hospital or Tama Nagayama Hospital, Japan.

CSF neopterin levels were significantly higher in 11 patients with febrile convulsions than in 22 with fever without convulsions or in 7 with convulsions without fever, 4 with status epilepticus. The CSF neopterin/serum neopterin ratio was also higher in patients with typical febrile convulsions, and those with prolonged febrile convulsions had higher CSF neopterin levels than patients with typical febrile convulsions. CSF interferon-gamma showed a tendency to higher levels in patients with febrile convulsions. (Kawakami Y, Fukunaga Y, Kuwabara $\mathrm{K}$ et al. Clinical and immunological significance of neopterin measurement in cerebrospinal fluid in patients with febrile convulsions. Brain Dev Oct 1999;21:458-460). (Respond: Dr Y Kawakami, Nippon Medical School Second Hospital, Department of Pediatrics, I-396, Kosugi, Nakahar-ward, Kawasaki, Kanagawa 211-8533, Japan).

COMMENT. Neopterin CSF levels are elevated in children with febrile convulsions, pointing to a mechanism involving an immune activation in the central nervous system. None had pleocytosis of the CSF, and the cause of the elevated neopterin levels is undetermined.

\section{LOW-DOSE ACTH THERAPY FOR INFANTILE SPASMS}

The lowest effective ACTH dose, with fewest adverse effects, for the treatment of West syndrome (WS) was determined in a comparative, randomly assigned, controlled study involving 25 patients with cryptogenic (CWS, n=9) or symptomatic (SWS, $\mathrm{n}=16$ ) WS, at the Department of Pediatrics, Tokyo Women's Medical University, Tokyo, Japan.

Either low dose $(0.005 \mathrm{mg} / \mathrm{kg}$ per day $=0.2 \mathrm{IU} / \mathrm{kg}$ per day) or high dose $(0.025 \mathrm{mg} / \mathrm{kg}$ per day $=1 \mathrm{IU} / \mathrm{kg}$ per day $)$ synthetic ACTH, was administered every morning for 2 weeks and tapered to zero over the subsequent 2 weeks. In the CPS group, infantile spasms and hypsarrhythmia were completely controlled in $3 / 4$ given low-dose and $5 / 5$ on high-dose ACTH. In the SWS group, spasms and hypsarrhythmia were controlled in $6 / 8$ at each dose level. No significant differences were observed between low and high-dose ACTH for either type of WS. Long-term responses in the 17 responders followed for more than 1 year showed no significant differences among groups. Sleepiness and brain shrinkage estimated by CT scan were significantly milder in the low-dose group. Low-dose ACTH therapy may be equally effective as high-dose and is recommended in CWS and in SWS with cerebral atrophy. (Yanagaki S, Oguni H, Hayashi K et al. A comparative study of high-dose and low-dose ACTH therapy for West syndrome. Brain Dev Oct 1999;21:461-467). (Respond: Dr Shigeru Yanagaki, Department of Pediatrics, Tokyo Women's Medical University, 8-1 Kawada-cho, Shinjuku-ku, Tokyo 162, Japan).

COMMENT. The controversy regarding the optimum dosage of ACTH for treatment of infantile spasms continues, the low-dose, short-duration regimen favored in Japan, the high-dose, extended treatment advocated by the majority in the United States and the UK. My own preference has been for smaller doses (1020 units Acthar gel daily IM for 3 weeks), with relative avoidance of serious side- 